\title{
SERVICE COMPOSITION BY USING LOAD AND MOBILITY IN OPPORTUNISTIC NETWORKS
}

\author{
Sathya A $M^{1}$, Shalini $M^{2}$, Shwetha G $S^{3}$, Manjusha Kulkarni ${ }^{4}$ \\ ${ }^{1}$ UG scholar, Computer Science and Engineering, T John Institute of Technology, Karnataka, India \\ ${ }^{2} U G$ scholar, Computer Science and Engineering, T John Institute of Technology, Karnataka, India \\ ${ }^{3} U G$ scholar, Computer Science and Engineering, T John Institute of Technology, Karnataka, India \\ ${ }^{4}$ Assistant Professor, Computer Science and Engineering, T John Institute of Technology, Karnataka, India
}

\begin{abstract}
Mobile devices carried by users have the capacity to make use of components in networks that provide services which can be combined to contribute the numerous application level services. As mobile devices are prone to disconnection periodically and partition his result in the necessity of opportunistic networking techniques to communication between the devices. Also this kind of scenarios posses challenges to service composition techniques. There are three phases in our proposed work:1)Selecting suitable set of service sequence;2) routing results of previous node to the next node providing service using multi-hop-path; And result is sent back to the node which is requesting for service. Even though, no direct connections occur between the nodes which are in mobility condition. The nearby services are accessed and given to the user, It can be implemented by combining many services. To combine these services following parameters are considered: "shortest distance", "load". Using this information, we estimate the combination of multiple services.
\end{abstract}

Keywords: Service Composition, Opportunistic Networks, Mobility Models

$* * *$

\section{INTRODUCTION}

In modernistic years, multi functionalersonal digital assistant has increased. Digital devices contact with other in the range increased by the use of many implant(embedded) proportion, in which such opportunistic connection between the digital devices are used in the network. Different resources on every devices are accessible can be absorbed as services and contain the independent platform services are put together from the different available services to increase the function [7],[8] or performance.

For example consider a scenario; event is taking place in college .other department student need the information about that particular event. Resources or information related to the event are available on every digital device drifted by people in existing surrounding, utilizing digital device in existing nearest to the event. By these information we create a event context. Event context are used to filter the related information, perform processing on the filtered resource if required and then final result is gathered according to the available resources on each individual device. Each function in this scenario is considered as a service that can be executed on a device.

Service composition and opportunistic technique is applicable in intelligent transportation, crisis management, healthcare, etc[8] and environments like malls ,parks ,streets, or other common place. When a mobile user device needs a resource which is not locally accessible, service composition can be applied. Presently, using these resources untidily in mobile devices which do not have all the functionalities locally or devices that depends on a existing infrastructure to get the services from the internet is not possible.

Service composition in opportunistic networks is attended with high risk. For example, (1) In internet the expected services might not be accessible (2) The content that are downloaded from the Internet consumes bandwidth either limited or costly (3)contact with Internet itself is difficult during signal corruption and in outlying area. produce an environment that is enriched with services is a major challenge, as it is difficult to get the services on every devices where there is no route occur between them. To choose set of service sequence, Proposed algorithm calculates temporal distance and load in a distributed manner. The feasibility of our solution is that there is no need of connected path between the device that provide services and device that request for the service.

\section{RELATED WORK}

In this paper gives the explanation of one and many services of current service are representation in the investigation. service representation: To explain the importance of composed service, the research has been done on the definition and ideology[10]. Composed services defined strictly in the requesting static composition [11],or depends on the request service in dynamic composition can be evaluated. In the environment alternate set of available services are combined together to providence the appropriate functions. Feasible combining of services are designed as "directed connected graphs" [7]. To discover and explore the service, we influence composition opportunistic in the work to represent the service. 


\section{Requesting Single Services:}

For execution of services in the opportunistic networks parsarula et al[5][6] explore advantageous policy for service

. At the level of replication single service result

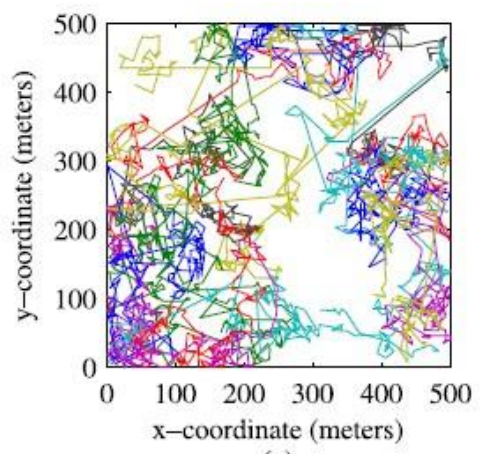

(a)

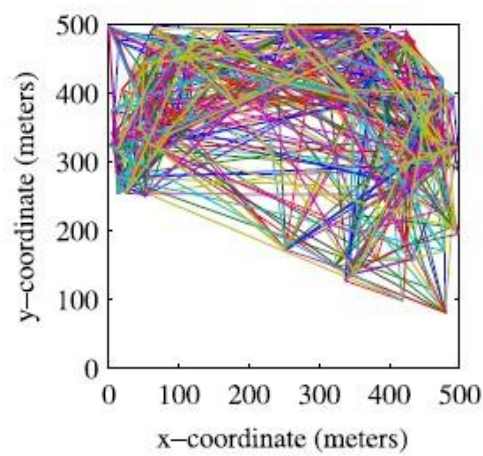

(b)

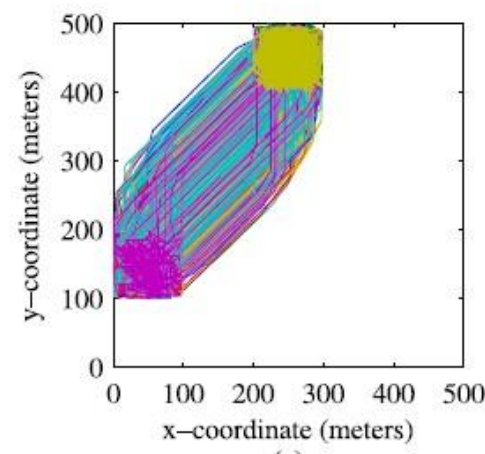

(c)

Fig.1 [1] Trajectories of 20 users in (a) Levy Walk, (b) SLAW, and (c) HCMM mobility models.

are received in less time and wait till the service requester meet the direct connect with service provider for the optimal policy and for discovering service multi-hop paths are disconnected for service, using set of proxies in the opportunistic networks[12]. However user location in this work, composition of many services are not consider. composing multiple services:

Forwarding mechanism does not discuss the number of architecture and middleware framework for service composition[7],[14]. Based on interaction of past by considering the time at location of node, use of multi-hop path send and receives in the intermediate node are considered. If nodes are not directly connected, service composition fails. In order to predict in future which node at what time it is connected, past interaction of node is required. In this paper even when an direct route does not occur in the service composition, using multi-hop path can pass the input service to service provider and gives the result service back to the requestor service. To increase the service composition alternative paths are considered.

\section{SYSTEM ELUCIDATION}

\section{1 "Service Model"}

To represent services, we work on a service graph based on input requirements such as concatenation of services, input and output. in a hierarchical manner[8]ideology and definition can be done by using services.i.e it can be composed of two services if one of output type is same as other type of input. For service possibility Spqand $\mathrm{Cd}$ input/output types, such that input type $p €[1, \mathrm{Cd}-1]$ and output type $Q €[p+1, x d]$.different level of services functionality are illustrated functionality of service $\mathrm{Spq}$ is $\mathrm{r}$, if $\mathrm{q}=\mathrm{p}+\mathrm{r}$, suppose $\mathrm{S} 23$ and $\mathrm{S} 34$ are service representation.

It does both "encryption" and "decomposition" represents the higher functionality of service. High service functionality such as S14 gives the same transformation otherwise with lower functionalities; by composing multiple services are possible. Varying service functionalities are demonstrate the accommodation between the exact service selecting (far distance network) versus smaller composition of services(available in local area of requested service). On the device time of execution are exponentially distributed to explain work and device heterogeneity nodes provide the number of services, hence the each service representation are varying, it can be explored in the service composition for performance.

\subsection{Mobility Model}

A numerical distribution[17] are assumed previously shown to consist Inter-contact Times (ICTs) for "power law distribution ".A numerical Inter-Contact Times[18] show random change in direction, nodes mobility representation in this paper are not used "power law distribution flight length and pause -time distributions[19] has been shows the traces of real mobility. we establish synthetic evidence using "Levy walk mobility "model[19] and "SLAW mobility" model[20] these are included in our simulation and analysis part too.

In "Levy walk" and "SLAW" more realistic scenario are represented where nodes meets each other evenly. By considering existing of relationship between the social user mobility are taken by using the "HCMM mobility "model[21].in additional its performance can be evaluated. In opportunistic network mobility model are evaluated and performs by using the wide range of its characteristics in service composition:"SLAW mobility" model-“clustered environment";"HCMM mobility" model - "movement in communities" and "Levy walk mobility" model -"mobile users are equally distributed". Also it plays a important role in forward and select the service request. Fig 1 levy walk uniformly distributed user mobility utilize the power law distribution by showing 20 trajectories of user this model. Under SLAW specific sites are visited (called way 
points [20]). This effects movement of users is uniformly distributed in 'levy walk'[fig 1a] and includes group of users in SLAW(fig 1b). Fig 1.c represents user movement only between two communities in which the movement is controlled by using the rewiring parameters of HCMM[21].

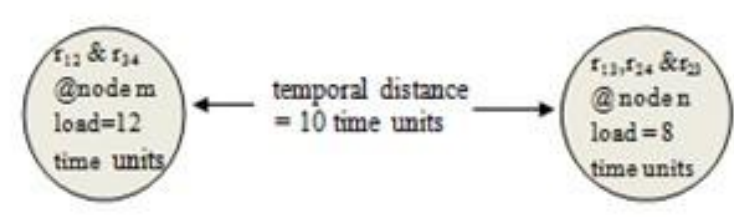

(a) Scenario of two nodes at some distance [selected path] $\left(r_{1}, m\right)$ to $\left(r_{13}, n\right)$ to $\left(r_{34}, m\right)$ to $\left(r_{4}, m\right): \cos t=40$ $\left(r_{1}, m\right)$ to $\left(r_{12}, m\right)$ to $\left(r_{23}, n\right)$ to $\left(r_{34}, m\right)$ to $\left(r_{4}, m\right): \cos t=52$ $\left(\mathrm{r}_{12}, \mathrm{~m}\right)$ to $\left(\mathrm{r}_{12}, \mathrm{~m}\right)$ to $\left(\mathrm{r}_{24}, \mathrm{n}\right)$ to $\left(\mathrm{r}_{4}, \mathrm{~m}\right): \cos \mathrm{t}=40$
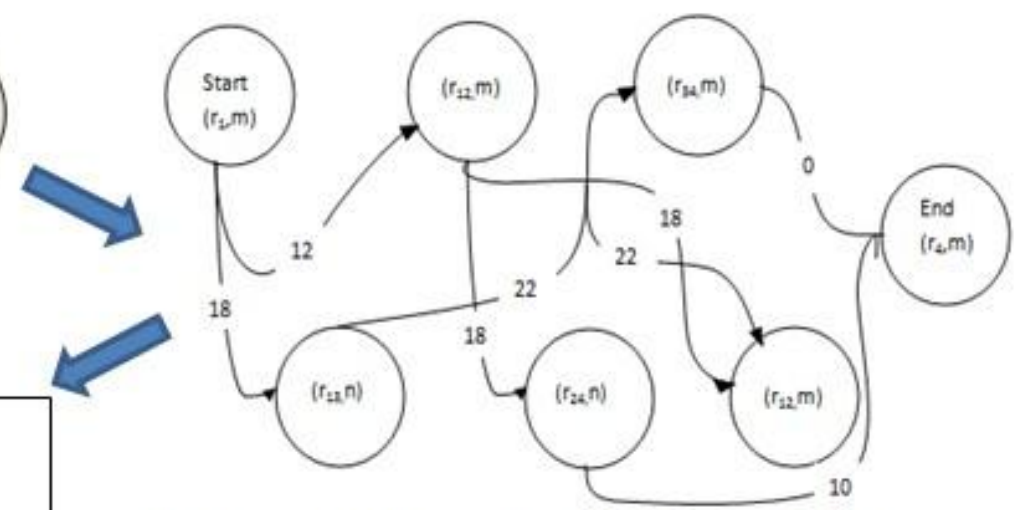

(b) Service Graph for request type 1 to output type 4

Fig. 2 [1]. Trajectories of 20 users in (a) Levy walk (b) SLAW and (c) HCMM mobility models

\subsection{Forwarding Schemes}

The forwarding schemes with good performance in dynamic environment are listed as i)using distance of node with similar characteristics in mobility or "time transivity"[3] ii) usage of mobility of nodes with different characteristics[3], "encounter rate(EBR)". In addition "modified version of timers(MT)"'[22] are proposed that is suitable for forwarding paths that are small. In service composition selection and forwarding mechanisms are compared based on four forwarding schemes-direct or one-hop, "TT","EBR", and "MT" in which "direct forwarding" comes under one-hop routing scheme and in multi hop routing schemes remaining forwarding schemes are categorized.

\section{SERVICE COMPOSITION ALGORITHM}

Overview of Service composition algorithm is described in this section. A particular service composition is selected based on the service graph which is maintained by each device and based on the service load and the temporal distance between the nodes, the link cost on the service graph is updated.

\subsection{Service Graph}

Based on the perspective of remaining network local service graph is maintained by each device which is represented as $\mathrm{S}=(\mathrm{P}, \mathrm{Q})$. Fig 2 represents a simplified case where service graph can be constructed by using service provided by two devices $\mathrm{m}$ and $\mathrm{n}$. The number of input/output types, devices and services (including recursion) are not, K, Respectively. Two types of vertices (A, B) are represented in service graph $V=\{A, B\}$, where vertex $c \in A$ is a service pair and device such that $|\mathrm{A}|=\mathrm{M}$, and another vertex $c \in \mathrm{B}$ is a input /output type pair and device $|\mathrm{B}|=\mathrm{n}$. These vertices of two kinds are represented in fig $2 \mathrm{~b}$. i)at node a , $(\mathrm{r} 12, \mathrm{~m})$ is a vertex of type A indicates the service provided r12. ii) at node a there is an entering/exiting and input/output application of type $1(\mathrm{r} 1)$ is $(\mathrm{r} 1, \mathrm{~m})$ of type B vertex . Maintainance of the service graph is at only vertices of types B.

A directed edge exists between vertex e and vertex $\mathrm{f}$ if(i) input service $c \in A$ is similar as input/output type of e $\epsilon B$. For example $(\mathrm{r} 1, \mathrm{~m}) \rightarrow(\mathrm{r} 12, \mathrm{~m})$; (ii) input service of $\mathrm{f} \in \mathrm{B}$ is same as output service e€Aeg., (r24,a) $\rightarrow(\mathrm{r} 12, \mathrm{~b})$ or (iii) input/output type of $f \in \mathrm{B}$ is similar as output services of e $\in$ Aeg., $(r 4, m) \rightarrow(r 24, n)$.In events (i) and (ii) the edge cost is calculated by the sum of the distance between the vertices at corresponding devices and the load at the second device vertex. Consider the example,Adition of shortest distance from $a$ to $b$ is 10 and $b$ load is 8 therefore, cost of $(\mathrm{r} 12, a) \rightarrow$ $(\mathrm{r} 24, \mathrm{~b})$ is 18 . But the egde cost in event(iii)is shortest distance of corresponding vertices at node. The shortest distance (a) from the hop requesting for service to the hop hosting the initial service (b) from the terminal service hop to requestor is considered by vertices of type $\mathrm{B}$.

\subsection{Algorithm}

Information about load on service, temporal distance and associate node provide services. service graph is created by a device, by using this information shortest path can be evaluated using our algorithm. At device a service request input type 2 to output type 4 can be explained in a simple case. on each device edge cost can be calculated from each service output is well matched to service inputs, by considering load on device and temporal distance between the nodes, Dijkstra's Algorithm is used to compute the shortest path from initial vertex $(\mathrm{r} 1, \mathrm{~m})$ to terminal vertex $(\mathrm{r} 4, \mathrm{~m})$ using final graph. requested service are routed from device $A$ to device $B$ by giving information to the forwarding algorithm. Services are executed in sequence, if device is holding service(device $n$ ) next available services are find, shortest path is re -computed for remaining request. Address of destination are given to forwarding 
technique. Final result using forwarding scheme can be routed back to the requester by finishing its re-computation and execution is done for all pending request. Networktopologies are feasible to provide the feasible path by re-computing and execution of each device service. Topology remains same for some duration it may have the chance of choosing same path for processing the service request. Contact between the two node data value $\mathrm{O}(\mathrm{N})$ can be shared .service graph has totally $\mathrm{M}+\mathrm{nt}$ vertices , these values are sum of load on device and temporal distance. service graph needs to updated on each device because edge cost remains same, the computational complexity of (edge cost) value as $\mathrm{O}(\mathrm{K} 2)$ instead of $\mathrm{O}((\mathrm{M}+\mathrm{nt}) 2)$.computational complexity of Dijkstra's algorithm. These algorithm finds service request composition paths have same kind of input type i.e from one input type to another type shortest path can be calculated .service request can be done number of times by using Dijkstra's algorithm.

\section{5. "RESOURCE MODELING AND PARAMETER ESTIMATION"}

\section{1 "Disconnected Paths"}

The distance between the devices that carried by the users is calculated as the nodes providing a source might not expected to be connected directly to the node requested for the service in opportunistic networks. The physical distance between nodes depends on the time separation(time elapsed between the nodes when they are not in each other's transmission range) is shown in [22]. The nodes can create a local view of the network by sharing the information about the separation in timei.e, the distance from each node in the network. To find and share values of separation in time, a method is given in [3] and the terminal value of separation in time which is calculated is the shortest temporal distance. To approximate the physical distance, several works have used the perspective insight of this idea 3],[22][24],[25],[26],[27]. Inspite of the relation between the separation in time and the physical distance, shortest temporal distance between two disconnected nodes is alternatively defined as the minimum time taken in which relay of information from one to the other is done by some sequence of contacts [27].

\subsection{Shortest Temporal Distance}

Significantly, the minimum time taken by some information to travel from node $\mathrm{u}$ to node $\mathrm{v}$ is provided by the shortest temporal distance(duv). The terminology temporal distance is always to shortest temporal distance in this paper. Therefore, by comparing the lower bound on the propagation time, we compare the efficiency of underlying forwarding schemes to send requests to different nodes in network. For example, by considering the distance of node $\mathrm{v}$ and $\mathrm{t}$ (duvanddut) from node $\mathrm{u}$, node $\mathrm{u}$ can either select the service provider as $\mathrm{v}$ or $\mathrm{t}$ based on which has smaller distance. To provide duv and dut we use the approximations dvu and dtu as they can be calculated at node $\mathrm{u}$ because there is no centralized infrastructure in opportunistic network and this is done by using simple timers in a distributed manner. The physical distance between nodes is related to temporal distance is shown in [22]. The temporal distance are approximately equal as the distance between $\mathrm{u}$ and $\mathrm{v}$ is same in both directions.

\subsection{Service Loads}

Node request for a service, associated device produce the service based on FIFO queue.one node need to initialize load on destination device to reduce the amount of time in service composition, Because service provider results as a busy even though service requester is directly connected to the service provider,done by sharing the load on every device.[section 5.4] in distributed way. Service load and shortest distance can be estimated at destination device are used by "selecting service algorithm".Service load can be predicted by using static mechanisms. current each node load is estimated as 1 and decaying factor as $\alpha$ of 0.5 .pending multiplied request and number of expecting request are used to calculate the current window load $l_{\mathrm{cw}}$. Estimation of updated load use the following conditions: $1=$ $\alpha \mathrm{ccw}+(1-\alpha)$ loldwindow time is $30 \mathrm{~s}$ and lold is the previous time window for load value.

\subsection{Estimation of Temporal Distance and Load}

In uniformly distributed, each node we evaluate shortest distance between the nodes .evaluate the distance of each intermediate node at some point ,time d0is become approximately equal to later accuracy of node time d0is not too long .by using below rule each device maintain a timer for every other node where uniformly distributed. Node $\mathrm{u}$ is made last contact with node $\mathrm{v}$, Letdm(u) represents the elapsed time(i) evaluated node $u$ at node $\mathrm{m}$ are denoted. Depending on each device time can be increased. Suppose node $\mathrm{m}$ and node $\mathrm{n}$ when they both connected to each other, their estimated timers and load are updated. When temporal distance of device $n$ is small defines as below: $\mathrm{Vu} \neq \mathrm{m}$ if $\mathrm{dn}(\mathrm{u})<\mathrm{dm}(\mathrm{u})-\mathrm{dmv}$,

Then

$\mathrm{dm}(\mathrm{u}):=\mathrm{dm}(\mathrm{u})+\mathrm{dmv}$

$\operatorname{Sm}(\mathrm{u}):=\operatorname{Sn}(\mathrm{u})$

If transmission range are within limited area distance between two nodes is denoted as tmv. The value of dmv small constant less than or equal to one unit and greater than zero i.e. $\operatorname{dmv} \epsilon(0,1]$.in our experiments physical distance value are not taken,instead small value of dmis used in experiments for time utilization.

\subsubsection{Service Advertisement and controlling}

\section{Distribution Radius}

"Service composition"algorithm evaluate shortest distance values by considering close values of each devices and also it keep track of each device .temporal distance value are maintained to obtain the "threshold frequency"how much delay can be controlled ,it may depends on the practical 
requirements. Node does the functionality of removing unnecessary information. size of network been increased and maintain a constant value for efficiently forwarding request and select.In network when two node come in contact with each other distance and load have been updated ,where threshold value become greater than distances.

\subsection{Levels of Awareness}

Our Algorithm compare with other forwarding scheme it stands independently particular service requesting shows effect on performance. Even though forwarding algorithm in service are used to transfer data efficiently but still we consider about the delivery of data.in the network bring the awareness of particular service selecting at different level. edge cost is estimated by using sum of distance and load on device .where first node a $\mathrm{Sm}(\mathrm{u})$ denotes load $\mathrm{du}(\mathrm{v})$, Temporal distance as du(v), only node a maintains time $\operatorname{dm}(\mathrm{u})$, awareness are used to estimate at different levels $\mathrm{du}(\mathrm{v})$ for $\mathrm{u}, \mathrm{v} \neq \mathrm{m}$.

In this case services are selected randombly.to lower level we all know that services are provided but do not contain a particular service distance value.

In the network every node knows latest node service, also has the information about local service which previously described in section 5.4.occurance of delay is possible when it is distributed uniformly. per connect level is in the need to exchange of values $\mathrm{O}(\mathrm{K}), \mathrm{dm}(\mathrm{u}), \mathrm{Sm}(\mathrm{u}) ¥ \mathrm{u}$ at node $\mathrm{m}$. each device need to know temporal distance to update the cost of devices.Anyway this information is not available in local knowledge .each device maintain their own timers. We can show local knowledge performances result is closely achieved with prefect knowledge. node a uses $(\mathrm{dm}(\mathrm{u})+\mathrm{dm}(\mathrm{v}))$ temporal distance approximation $\mathrm{du}(\mathrm{v})$ because $|\operatorname{dm}(\mathrm{u})-\mathrm{dm}(\mathrm{v})|<\mathrm{du}(\mathrm{v})<\mathrm{dm}(\mathrm{u})+\mathrm{dm}(\mathrm{v})$. Receive node are uniformly distributed each device consists of timer for distributed knowledge.

\section{PERFORMANCE EVALUATIONS}

In our proposed solution without using service composition, we are going to compare the performance solution to demonstrate the effectiveness. When service request are created in multiple hob path where services are pass simultaneously, by using service composition in other device node. Exact match is not possible in opportunistic network. Does not explain about usage of opportunistic network but composition have been taken. Service request rate have been reduced by service concept in opportunistic network. For next remaining network, local knowledge have been maintained to achieve good result (section 6.3) comparing by several mechanism (section 6.5) and four version of our proposed solutions are evaluated.

To improve our performance we divide the service 1, service 2 to observe how far service request have been reached in multiple path.(section 6.4) hop count is distributed to observe high load effect,time duration of long request and service node destinations..(Section 7.1) current load information about advantageous.Exact solutions with respect to degree of approximation (section 7.3) .

Mobility characteristics are evaluated by using synthetic traces in environment to control.by logging GPS we can track and trace behavior of mobility.in our paper identifying the mobile user opportunities are analyzed.

\subsection{Simulation Setup}

Users carry the GPS to login the position at $30 \mathrm{sec}$ and there interval in a state fair [28],information about mobility have been collected.to compare the performance of user like track logs and number of nodes on different days are taken as separate user on the same day. every day it has duration of eight to ten hours in a day to login. We cut all user login to 90 hours 18 users locations are recorded. "Levy walk "generates synthetic mobility traces.

All minimum and maximum are run; error respectively shows five simulations can be plotted. Table 2 summarizes simulation parameter. In forwarding scheme "EBRencounter rate window" $=10 \mathrm{~min}, \mathrm{dmv}=10 \mathrm{~min}$ for time transitivity and 0.5 for modified mobility model are given as parameter .after 15 min service request will get out, to avoid the overloading of services. suppose service is at level 3 in repetition level, exact 3 services are provided with unique id. Total average time is $30 \mathrm{sec}$ and generates the services with $\mathrm{z} \geq 4$. services are not proceed when not task is not completed within $15 \mathrm{~min}$, last $30 \mathrm{sec}$ request cannot be occurred. Transient regime and steady regime phases are included in simulation.

Initially at transient state does not with service load till 30 min service lasts approximately, after collecting information about network it reach to steady state. Composition of delays are exists in steady state.

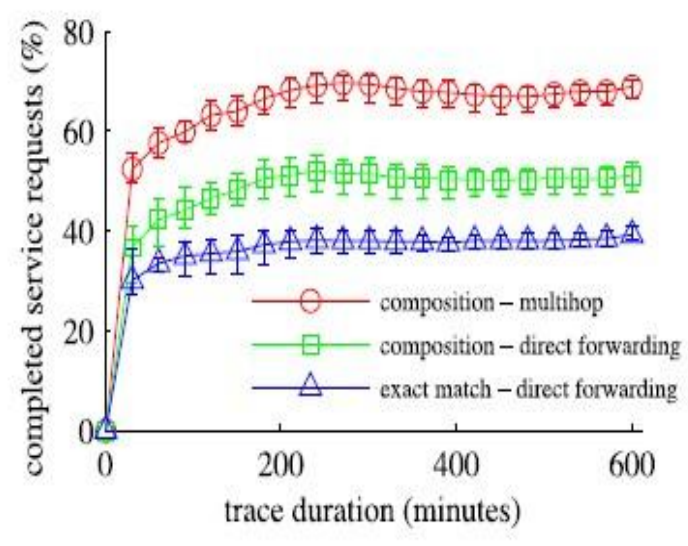

Fig.3 [1] Higher service composition in multi-hop composition

\subsection{Direct and Multi-hop Forwarding}

Service graph can be constructed by using local level of awareness and completed in Levy walk. multiple service composition service try to meet the 50 percentage of 
requirements. Forwarding services and output in multi hop path about 70 percentage is completed. multi hop forwarding scheme can be achieved in service composition efficiently compare with others[15],[16].

Delays are more in one hop path compare to multi hop path in opportunistic network, following fig.4 represents it takes 5 min delays in opportunistic network, where as direct one hop consumes 15 min. Hence we can conclude delays are minimized by using composition service in opportunistic network.

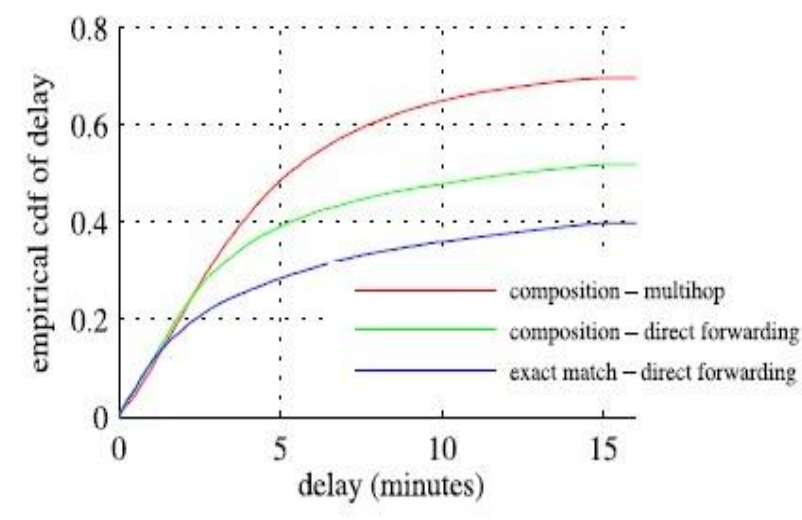

Fig.4 [1] Lower delay in multi-hop composition

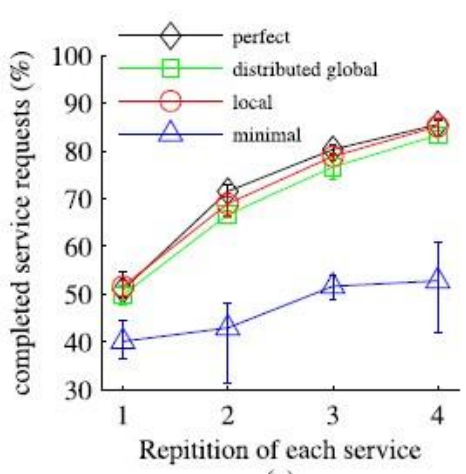

(a)

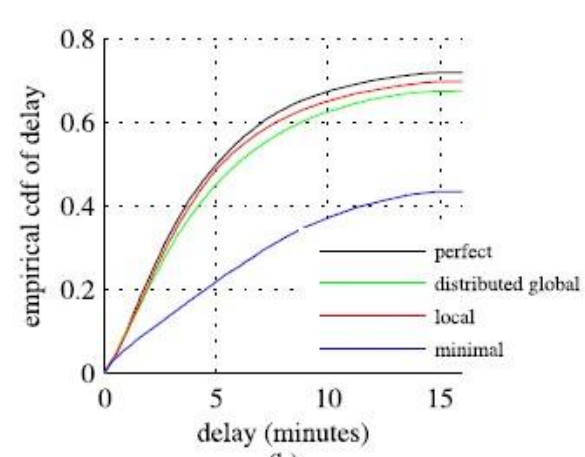

(b)

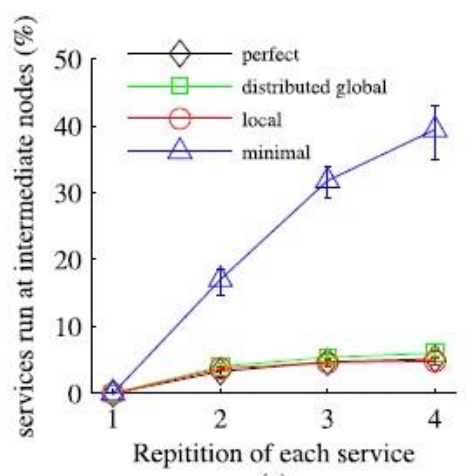

(c)

Fig .5 [1] Levy Walk :With different levels of awarenes (a) service completion, (b) delay, and (c) services run at intermediate nodes

\subsection{Levels of Awareness}

Service composition signifies best improvement by knowing information about load and temporal distance.fig.5a denotes perfect range in completion, destination service and within 3 percentage local awareness to each other. in fig.5b two different device gives same service, repeated twice and increase the local ,global delay in this figure. Local knowledge has been use to estimate distance of other device for selecting service. during the time of forwarding scheme in network topology, received services does not make much changes in it. In fig 5.c when service come in contact with each other there different service density, percentage can be increased by selecting device correctly in service composition.

\subsection{Composition Length, Delay, and Hop Profiles}

Levy walk Mobility traces and state fair are detailed in service composition. Duration of Levy walk consumes 10 hour to estimate result in steady state, where as fair trace lasts 90 minutes.

Every node in network provide at least one service and degrade the performance in exact match.fig .7a shows service completes in higher rate and minimal delay. Using less hop less than 5 completes service composition in fig 7b.when service requesting if nodes are not available it takes at least two hop.fig .7c empirical CFD service takes time to request and return back the result to requester node .service request have $10 \mathrm{~min}$ less delay in 60 percentage of completed service request.

\subsection{Effect of Forwarding Scheme, Request Load,}

\section{Request Timeout, Node and Service Density}

Service selection algorithm are used in forwarding scheme to show completion rate of service under 3 different level, transform the single copy of data, using different connectivity level and service load are analyzed in network performance, in fig.8a At high node/service density decreases the service are completed within duration of time out[fig.8b].any changes are occur under $5 \mathrm{~min}$ as shown in [fig.8a]. Therefore higher loads follow in extended service queue and services make use of lengthy execution, when a service provider are connected path, there is an distinct in request time out .modifies the delay view at over $10 \mathrm{~min}$ as shown in fig. 8 b. Since longer request time outs maintain the service request in queue, if not it is rejected without completion. As a result the service load at nodes to improve marginally. 


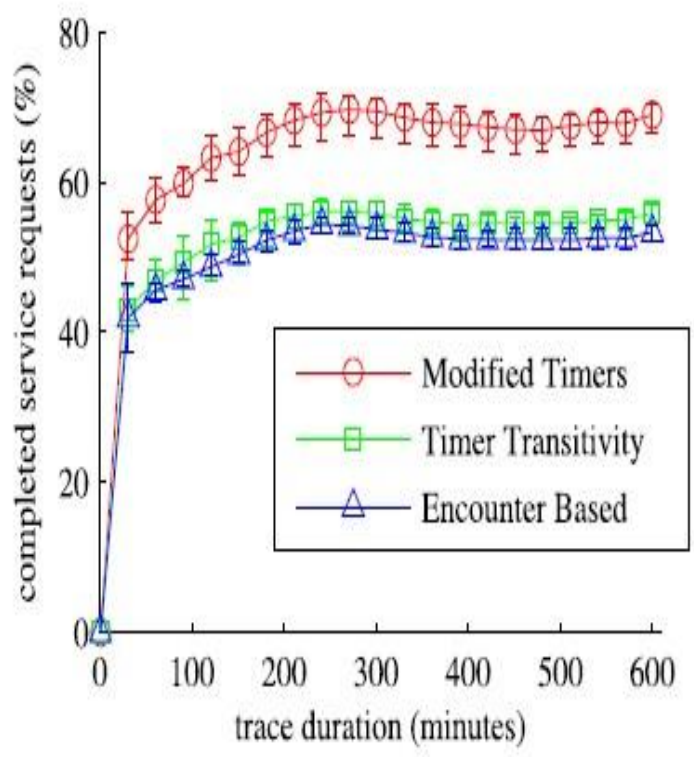

Fig.6 [1] MT with better service completion.

\section{CHARACTERISTICS OF THE ALGORITHM}

Performance in distinct mobility characteristics: evenly speeded, groups and communities is show the key features by which the performance have been improved or to show the limitations while the service composition improves its performance in opportunistic networks. Impact of mobility is evaluated by changing the parameters of mobility. The difference in the cost of combining services are computed in the service plot and exact cost to completion is examined to evaluate the performance variation in various mobility environments. The correctness of the shortest distance path is in directed by this difference of composition cost as a way of estimating the accessibility services.

\subsection{Mobility characteristics}

The accomplishment of services combined with varying mobility parameters in "Levy walk", "SLAW" and "HCMM mobility" models is shown in fig. 9. In "Levy walk" and Slaw, the length of flight and distribution of time are similar

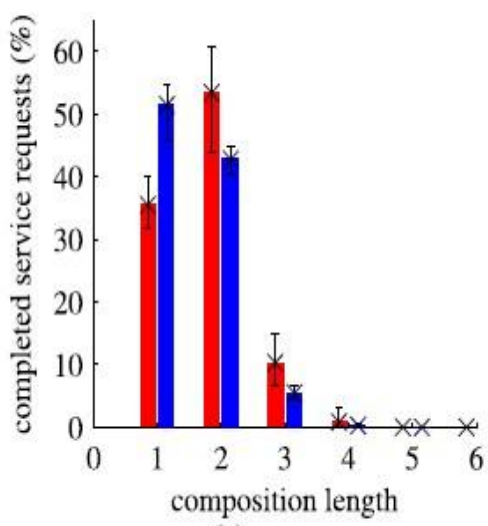

(a)

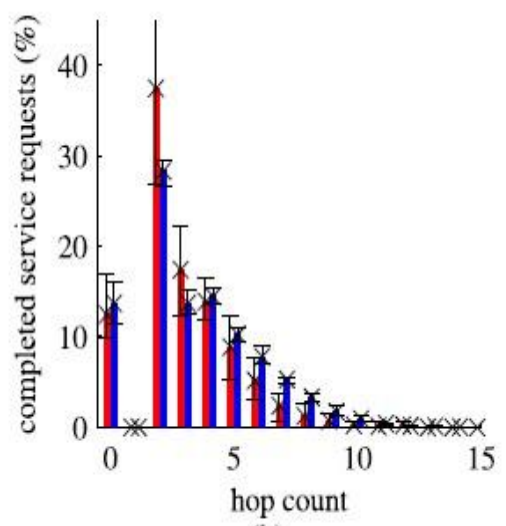

(b)

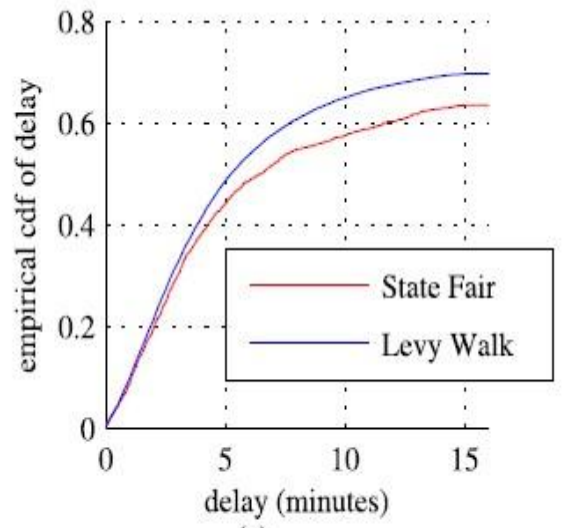

(c)

Fig .7 [1] state Fair[red] and Levy Walk [blue] mobility traces: (a) composition length, (b) hop count, and (c) delay profile for multi-hop service.

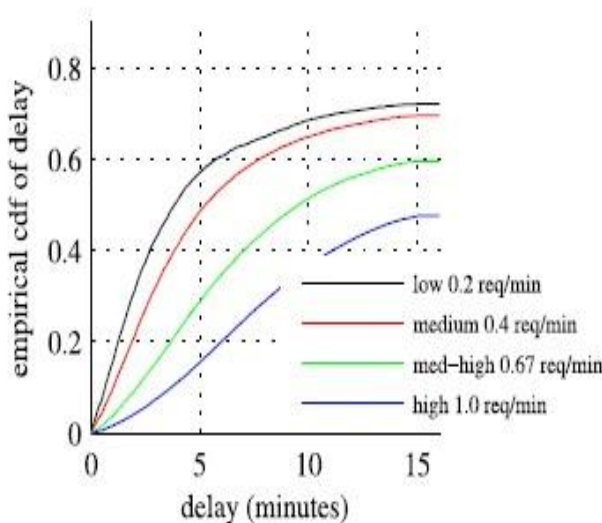

(a)

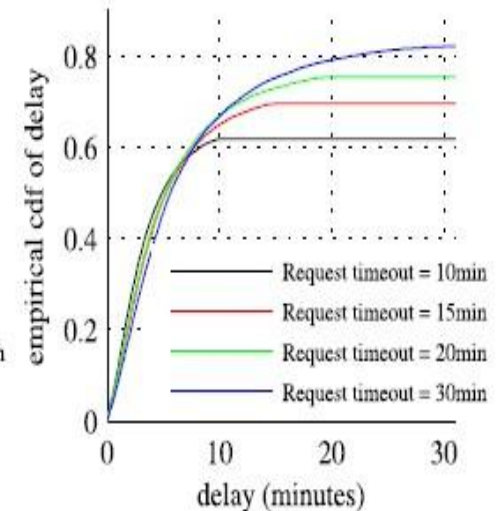

(b)

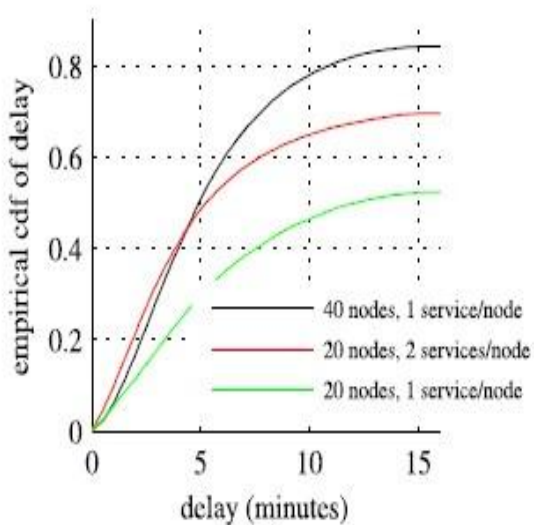

(c)

Fig .8 [1] Levy Walk: Higher service completion and lower delay at (a) low loads, (b) long request timeout, and (c) high node, service density for multi-hop service composition. 
so those parameters are not varied. The parameter of hurts is used in "SLAW" to control the degree of grouping of nodes in environment. Also the HCMM has key distinctive feature Decreasing density of user and service completion rate is shown in fig. 9a; For example when increases the area of simulation from $500 \mathrm{~m} \times 500 \mathrm{~m}$ to $900 \mathrm{~m} \times 900 \mathrm{~m}$, the service completion in HCMM varies from 81 to 64 percent. Effect of Hurst parameter is shown fig. $9 \mathrm{~b}$ which significant at lower user density, the performance of service composition decreases. We use Hurst parameter value 0.75 in performance comparison of SLAW, Levy walk and HCMM tracing of real world have this value[20],[29].

The result of probability in" HCMM" is shown Fig.9c. When rewiring probability increased from zero ,initially there is a decrease and then increase in accomplishing services because when rewiring probability $=0$, every nodes are staying in same community thus more services are completed versus when rewiring probability $=0.1$, before completion of pending requests some of nodes leave. Increase in rewiring probability results in improvement in percentage of completed service.

\subsection{Load Estimation}

Combining multiple service in algorithm is examined by considering load and without load. 1)"LA" : Service composition conscious with load (algorithm makes use of temporal distance and load at other nodes) 2) "NLA": Service composition without the conscious of load(algorithm makes use of only temporal distance )

Fig. 10 display service composition conscious with load(LA). In a huge connected network it increases the performance by $10 \%$, except it do not create a lot of changes in scattered connected networks in HCMM mobility. When devices are connected to each other in network performance of LA increases than NLA in huge connected networks, since connection are lightly scattered compared with delay to load on each device. Performance of LA is identical to NLA is occur in scattered networks. Brings awareness of load and give importance to ,which services to be composed. As a result, the necessity to retain trace of load at each nodes is critical because late in queuing and service execution request is comparable to forwarding delays.

\subsection{Estimated Cost and Actual Delay}

Based on the estimated time required to complete service, the accuracy of the values are analyzed in this section. It is shown by figure.1.1, the calculated cost for 70 to 80 percent of services which are completed in all three models lies in range of $4 \mathrm{~min}$ of real delay Also, for 40 percent of completed services, the estimated cost lies within 2 minutes of actual delay in all three models. This proves that to complete the request by estimating and selecting the optimal services that can be composed using distributed "Service Composition algorithm" with 'local' knowledge. Fig. 12 displays in Levy walk, evaluate cost of services that are not completed and is appropriate by 50 percent apart from
"SLAW" and "HCMM" is inaccurate. In order to measure the temporal distance is not suitable when the user movement contains body of clusters and communities except when user movement is continuously distributed in the surrounding area has agreeable accuracy. Except in HCMM and SLAW the nodes forget the community at random which results in inaccuracy hence services are incomplete in greatest number.

\section{CONCLUSION}

An algorithm for "service composition in opportunistic networks" is proposed to enable the users in mobility to make use of set of services to accessible in near place.

The perception of our solution is that the accessibility of nodes can be provided by estimating the temporal distance even though direct route never exists between the nodes. Considering service graph, order of composed service can be selected to accomplish the services required by the service requester and the forwarding service parameters can be done by any routing techniques.

\section{REFERENCES}

[1]. V.Sadiq ,M.Kumar, A.Parsella and M.Conti, "Service Composition in Opportunistic Network: A Load and MobilityAwareSolution" Trans.Comput.,vol.64,no.8, pp. 2308-2322 Aug.2015.

[2]. L. Pelusi, A. Passarella, and M. Conti, "Opportunistic networking:Data forwarding in disconnected mobile ad hoc networks," IEEE commun.Mag., vol. 44, no. 11 pp. 134141, Nov.2006

[3]. T.Spyropoulos,K.Psounis,C.S Raghavendra,"Efficient routing in intermittently connected mobile networks: The single copy case,'IEEE|ACM Trans.Netw.,vol.16,no.1,pp.63-76,Feb.2008.

[4]. S.Nelson,M. Bakht,and R. Kravets,'Encountered based routing in DTNs,"inproc.IEEE Conf .Comput.Commun.,2009,pp.846-854.

[5]. A.passarella, M. Conti, E.Borgia ,and M.Kumar,"Performance evaluation of service execution in opportunistic computing network," in Proc. 13th ACM Int. Conf. Modeling,Anal. Simul. Wireless Mobile Syst.,2010, pp.291-298.

[6]. A.passarella, M. Kumar, M. Conti, and E.Borgia, "Minimum delay service provisioning in opportunistic network," IEEE Trans. Parallel Distrib.Syst.,vol. 22,no.8,pp. 1267-1275,Aug.2011.

[7]. S. Kalasapur, M. Kumar, and B. A Shirazi, "Dynamic Service composition in pervasive computing," IEEE Trans. Parallel Distrib. Sys.,vol. 18, no. 7,pp.907-918,jul.2007.

[8]. M. Conti and M. Kumar, "opportunistic in opportunistic computing," computer,vol. 43,no. 1, pp.4250,Jan.2010

[9]. U.Sadiq,MKumar,A.Passarella, and Conti,’Modelling and Service Composition in Opportunistic networks," in Proc. 14th ACM Int. Conf. Modeling, Anal. Simul. Wireless Mobile Sys.,2011,pp.159-168. 
[10]. D. Chakraborty, A, Joshi, T. Finin, and Y,Yesha,"Service composition for movile environments," Mobile Netw. Appl., vol. 10,pp. 435-451,2005.

[11]. X. Gu, K. Nahrstedt, and B. Yu, "Spidernet: An intergrated peer-to-peer service composition framework," in proc. IEEE Int. Symp.HighPerform.Disturb. comput.,2004,pp.110-119.

[12]. N. Le Sommer and R. Said, and y.Maheo," A Proxybased model for service Provision in opportunistic networks," in proc. 6th Int. Workshop Middleware Pervasive Ad-hoc Comput.,2008,pp.7-12.

[13]. N.L. Sommer and S. B. Sassi,"Location-based service discovery and delivery in opportunistic networks," in proc. 9th Int. conf.Netw., 2010,pp. 179-184.

[14]. O. Davidyuk, I. Sanchez, and j. Riekki, "CADEAU: Supporting automatic and user-controlled application composition in ubi-quitous environments," Pervasive Comput.Commun.Des. Deployment: Technol.,Trends,Appl.,pp.74-102,2010.

[15]. L. Del Pretye and L. Capra, "Reliable discovery and selection of composite service in mobile environments," in proc IEEE 12th Int. Enterprise Distrib.ObjectComput. Conf.,2008,pp. 171-180.

[16]. J. Wang,"Exploiting mobility prediction for dependable service composite in wireless mobile adhoc networks," IEEE Trans.Serv.Comput.,vol. 4,no.1, pp.4455,jan.-mar.2011.

[17]. A. Chaintreau, P. Hui, J. Crowcroft,C. Diot,R. Gass, and J. Scott "Impact of human mobility onopportunistic forwarding algorithms," IEEE Trans.Mobilecomput.,vol. 6,no.6,pp. 606-620,jun.2007.

[18]. G.Sharma and R. Mazumdar,"Scalling laws for capacity and delay in wireless ad hoc networks with random mobility," in proc. IEEE Int.Conf. Commun., 2004,pp.3869-3873.

[19]. I. Rhee,M. Shin, S. Hong, K. Lee, and S.Chong,"onthev Levy-walk nature of human mobility," in proc. IEEE conf. comput.commun.,2008,pp. 924-932.

[20]. K. Lee, S. Hong, S. J. Kim, I. Rhee, and S. Chong, "Slaw: A new mobility model for human walks," in proc .IEEE conf. comput.commun.,2009,pp.855-863. [21] C. Boldrini and A. Passarella,"HCMM: modeling spatial and temporal properties of human mobility deiven by users social relationships," comput. Comm.,vol. 33, no .9,pp.1056-1074,2010.

[22]. U. Sadiq and M. Kumar,'ProxiMol: Proximity and mobility estimation for efficient forwarding in opportunistic networks," in proc.IEEE int. conf.Mobile Ad-Hod Sensor Sys.,2011,pp. 312-321.

[23]. T.Spyropoulos, T. Turletti, and K. Obraczka,"Routing in delay tolerant networks comprising heterogeneous node populations," IEEE Trans.Mobilecomput.,vol.8,no. 8 pp.1132-1147, Aug. 2009.

[24]. H. Cai and D. Y. Eun,"Aging rules: What does the past tell about the future in mobile ad-hoc networks?" in proc. 10th ACM Int.Symp. Mobile Ad Hoc Netw. Comput.,2009,pp. 115-124.

[25]. H. Dubois-Ferriere, M. Grossglauser, and M.Vettwerli," Age maters: Efficient route discovery in mobile ad hoc networks using encounters ages," in proc. 4th ACM Int. Symp. Mobile Ad Hoc Netw.Comput.,2003,pp. 257-266.

[26]. M. Grossglauser and M. Vetterli," Locating nodes with EASE: Last encounter routing in ad hoc networks through mobility diffusion," in proc. IEEE conf.commun.,2003,pp.1954-1964.

[27]. J. Tang, M. Musolesi ,C. Mascolo, and V.Latora,"Chacracterising temporal distance and reachability in mobile and online social networks," SIGCOMM comput. Commun. Rev., vol. 40, no.1,pp.118124,2010 .

[28]. I. Rhee, M. Shin, S. Hong, k. Lee, S. Kim, and S.Chong(2009,jul).CRAWDAD trace [online].Available: http://crawdad.cs.dartmouth.edu/ncsu/mobilitymodels/GPS/ NC_State-Fair.

[29]. (2009).[online].Available:http://fif.Kr/fisc2009/doc/K hlee.pdf.

\section{BIOGRAPHIES}

Sathya A M, BE, Department of CSE T.John Institute of technology Bangalore

Shalini M, BE, Department of CSE T.John Institute of technology Bangalore

Shwetha G S, BE, Department of CSE T.John Institute of technology Bangalore

Manjusha Kulkarni, Assistant Professor, TJIT Bangalore. 